\title{
Silicon Oxynitride based photonics
}

\author{
Kerstin Wörhoff, Edwin Klein, Gamar Hussein, Alfred Driessen \\ University of Twente, Integrated Optical MicroSystems group, MESA+ Research Institute for Nanotechnology, \\ P.O. Box 217, 7500 AE Enschede, The Netherlands \\ e-mail: K.Worhoff@ewi.utwente.nl
}

\begin{abstract}
Silicon oxynitride is a very attractive material for integrated optics. Besides possessing excellent optical properties it can be deposited with refractive indices varying over a wide range by tuning the material composition. In this contribution we will summarize the key properties of this material class and discuss several application examples. Preliminary results on novel processes, which will lead to largely reduced hydrogen incorporation and enable reflow of SiON material, are being presented.
\end{abstract}

Keywords: Silicon oxynitride, silicon nitride, chemical vapor deposition, integrated optical devices, hydrogen reduction, annealing

\section{INTRODUCTION}

The challenges posed by the continuously increasing information flow generated by modern electronic data processors can only be met by the application of optical techniques. Also in other fields like health-care or sensing the potential of optical solutions are being recognized. Consequently, increasing attention is paid to the development of reliable, low-cost fabrication processes for the realization of integrated optical telecom and sensing devices. Silicon oxynitride belongs to the favored materials, because it combines excellent optical properties with $\mathrm{Si}$-compatible fabrication techniques. SiON is especially attractive as it is transparent in a broad range of wavelengths from 210 to beyond $2000 \mathrm{~nm}$. The tunability of the refractive index $n$ over the wide range from $1.45\left(\mathrm{SiO}_{2}\right)$ to $2.01\left(\mathrm{Si}_{3} \mathrm{~N}_{4}\right)$ allows for a high flexibility in optical waveguide design [1]. This means, the channel waveguide and mode geometry can be adjusted from low-contrast designs for fiber-mode-field matching to high contrast waveguides applicable in very compact devices with large integration density. Deposition, mostly on thermally oxidized Si-wafers, is carried out with CMOS compatible deposition processes like Chemical Vapor Deposition (CVD). Numerous devices based on silicon oxynitride or silicon nitride, grown by Plasma-Enhanced (PE)CVD or Low-Pressure (LP)CVD, respectively, have been realized and successfully demonstrated [2]-[6].

\section{RESULTS}

At our institute, the PECVD and LPCVD grown thin films are prepared using an Oxford Plasmalab System 133 reactor and a Tempress deposition furnace, respectively. An overview of layer and waveguide properties based on silicon nitride and a selected silicon oxynitride composition can be found in table 1 . As can be seen, the materials can be grown reproducibly at sufficiently high deposition rates and yield excellent uniformity.

Table 1: Overview of properties of LPCVD silicon nitride and PECVD silicon oxynitride thin films.

\begin{tabular}{|l|l|l|}
\hline Parameter & LPCVD Si $_{3} \mathrm{~N}_{4}$ & PECVD SiON \\
\hline deposition rate $\mathrm{R}[\mathrm{nm} / \mathrm{min}]$ & 4.9 & 45 \\
\hline thickness non-uniformity $\delta \mathrm{d}[\%]$ & 0.6 & \pm 1 \\
\hline reproducibility of thickness [\%] & 0.7 & \pm 1 \\
\hline refractive index $\mathrm{n}_{\mathrm{TE}}$ & $2.0115^{*}$ & $1.5325^{\#}$ \\
\hline refractive index $\mathrm{n}_{\mathrm{TM}}$ & $2.0027^{*}$ & $1.5346^{\#}$ \\
\hline material birefringence $\Delta \mathrm{n}_{\mathrm{TM}-\mathrm{TE}}$ & $-8.8 \times 10^{-3}$ & $2.1 \times 10^{-3}$ \\
\hline refractive index non-uniformity $\Delta \mathrm{n}$ & $<5 \times 10^{-4}$ & $\pm 3 \times 10^{-4}$ \\
\hline reproducibility of refractive index & $<5 \times 10^{-4}$ & $\pm 3 \times 10^{-4}$ \\
\hline optical loss $\alpha[\mathrm{dB} / \mathrm{cm}]$ & $<0.2^{*}$ & $<0.2^{*, \#, \ddagger}$ \\
\hline
\end{tabular}


An example of a thermally tunable micro-ring resonator in $\mathrm{Si}_{3} \mathrm{~N}_{4}$-technology is shown in figure 1 . In this technology micro-ring resonators with a radius down to $25 \mu \mathrm{m}$ have been realized and tested [7]. The response upon thermal tuning of an $\mathrm{Si}_{3} \mathrm{~N}_{4}$-ring-resonator device with a $50-\mu \mathrm{m}$ radius can be seen in figure $1 \mathrm{c}$ ). The power consumption for the wavelength tuning in the drop channel is $10-20 \mathrm{pm} / \mathrm{mW}$ and the switching time is between 0.1 - 1 ms.

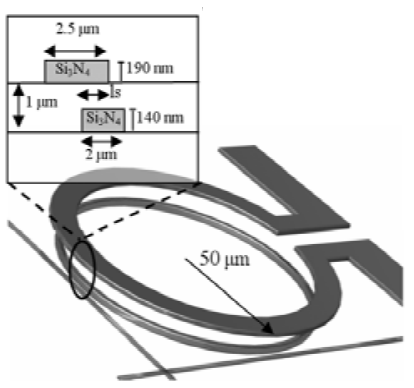

a)

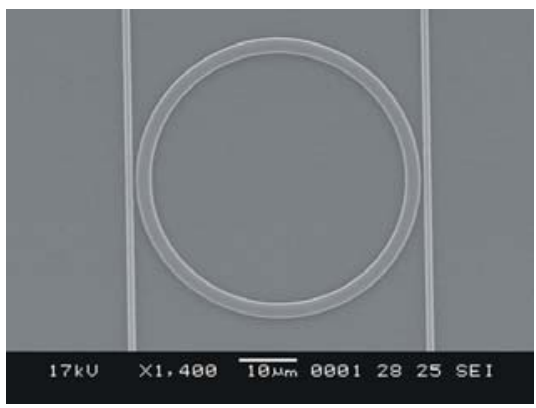

b)

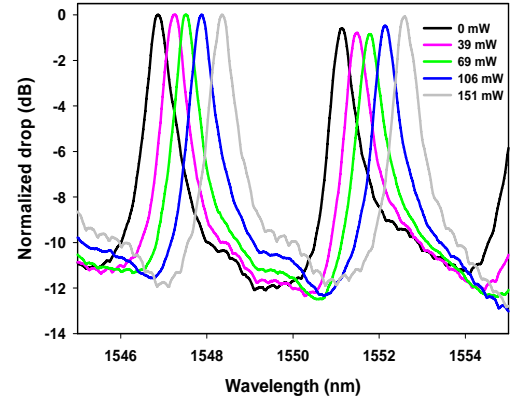

c)

Figure 1: Thermally tunable micro-ring resonators in silicon nitride technology: schematic layout (a), SEM micrograph of fabricated ring resonator (b), measured drop response for 0-150 $\mathrm{mW}$ applied power(c)

The design and performance of a micro-ring-resonator based device realized in SiON technology is depicted in figure 2 [8], [9]. The depicted reconfigurable add-drop multiplexer (ROADM) has been designed for 4.2-nm free spectral range. In a 4-single-channel configuration where the MRs are tuned so that they each drop one of 4 adjacent channels (100 GHz ITU-Grid) the on/off resonance attenuation per channel is $-12 \mathrm{~dB}$ and the power consumption $\sim 446 \mathrm{~mW}$. The device can be re-configured within $1 \mathrm{~ms}$ to combined channel operation; then the on/off resonance attenuation per channel is enhanced to $-30 \mathrm{~dB}$ and the tuning power drops to $20 \mathrm{~mW}$. Based on this OADM layout a 4-wavelength WDM router has been realized by combining four ROADM's.

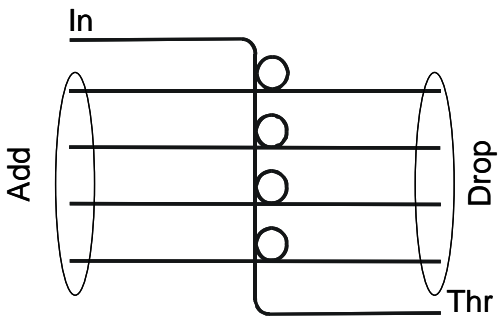

a)

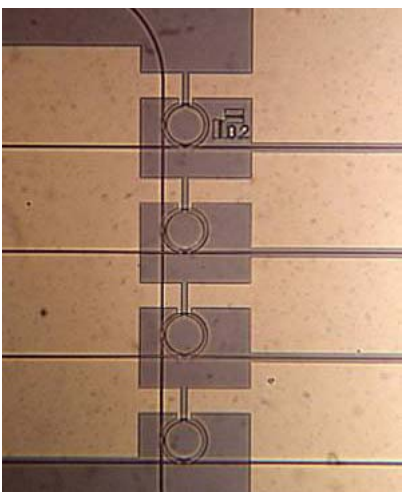

b)

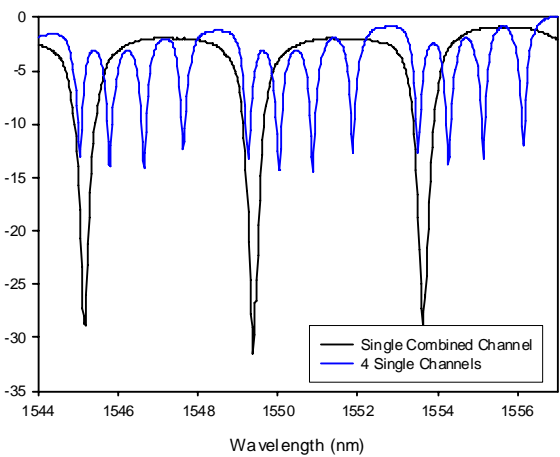

c)

Figure 2: Reconfigurable add-drop multiplexer based on ring resonator devices in SiON technology: (a) schematic layout, (b) realized device, (c) measured response.

The performance of the above described devices demonstrates the high potential of SiON technology in integrated optics. On the other hand, mainly for applications in the third telecommunication window the drawback of hydrogen incorporation in the SiON thin films becomes evident [10]. The vibrational overtones of the $\mathrm{Si}-\mathrm{H}$ and $\mathrm{N}-\mathrm{H}$ bonds cause absorption losses in the 1500-nm wavelength range. In general incorporation of hydrogen bonds is inherently due to the hydrogen-containing precursors of the deposition process and can only be effectively removed upon annealing at high temperatures $\left(1150^{\circ} \mathrm{C}\right)$. Treatment at high temperature causes, however, undesired changes in the material as well, e.g.: refractive index change, shrinkage, interface diffusion, and in the worst case cracks in the layer. Studies have been carried out recently with the goal of decreasing the hydrogen content in the as-deposited SiON layers and lowering the annealing temperature. From earlier research on BPSG deposition, it is known that incorporation of boron and/or phosphorus in silica decreases the melting point and allows for reflow at temperatures as low as $800^{\circ} \mathrm{C}$. We have carried out preliminary investigations on the boron and phosphorus doping in SiON thin films. It could be shown that similar to BPSG, also BP-SiON films can be reflown at reduced temperatures (see figure 3). This means that reflow of SiON waveguides and 
overgrowth material can be applied for extremely low-loss channel waveguides and void-free spacing layers, respectively. Furthermore, the hydrogen incorporation, annealing behavior and optical loss in phosphorus doped silicon oxynitride have been studied [11]. As it can be seen from figure 4a), the hydrogen content of SiON thin films decreases with the addition of phosphorus to the deposition process. From annealing studies it became evident that mainly the reduction of $\mathrm{N}-\mathrm{H}$ bonds is enhanced at lower annealing temperatures (figure 4c). The loss spectra of doped and undoped SiON waveguides have been measured after annealing at $1000^{\circ} \mathrm{C}$ (figure $4 \mathrm{~b}$ ). It can be clearly seen that the hydrogen-absorption peak around 1500-nm wavelength is completely reduced in the doped waveguides, while residual absorption is still present in the undoped case.

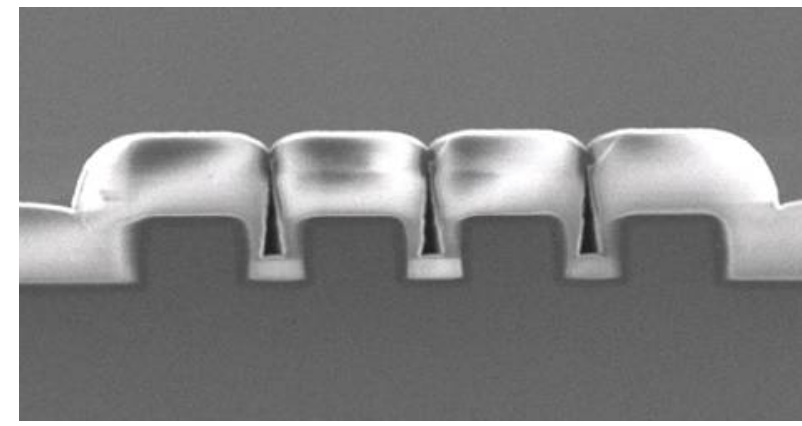

a)

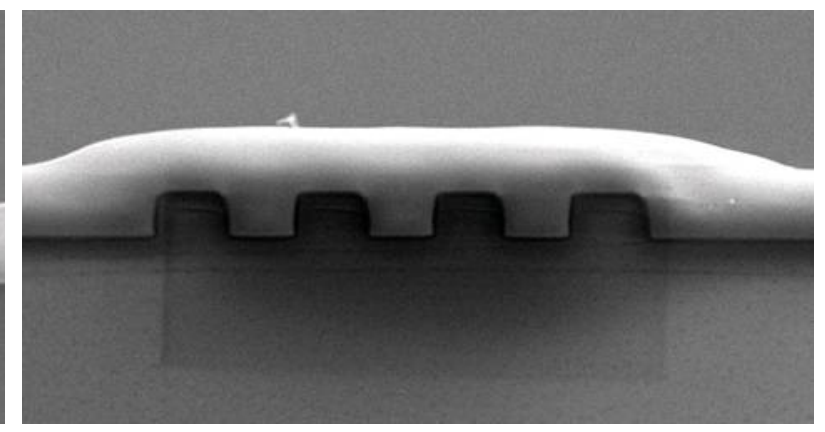

b)

Figure 3: Reflow of boron - phosphorus doped silicon oxynitride thin films grown on a patterned silicon wafer: as deposited (a) and after temperature treatment (b).

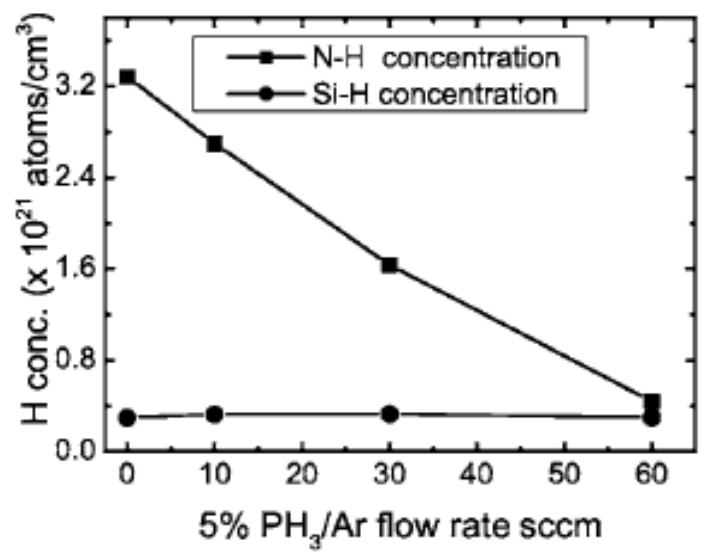

a)

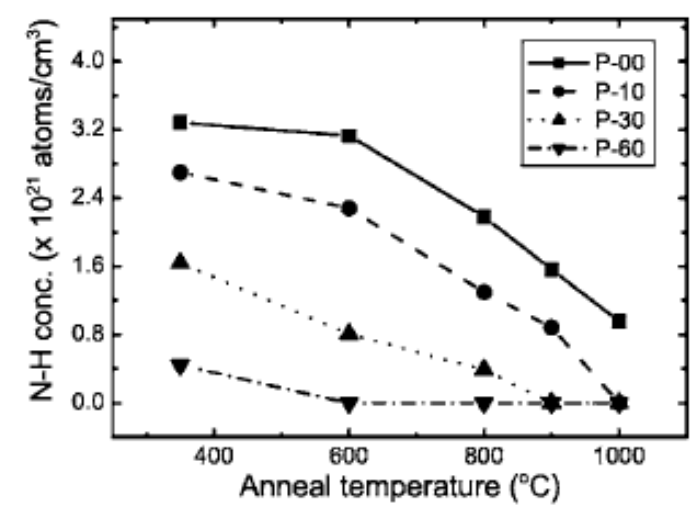

c)

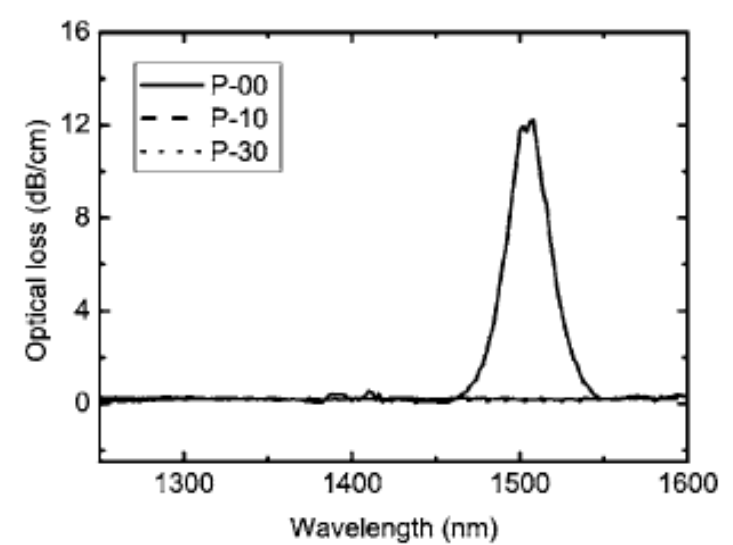

b)

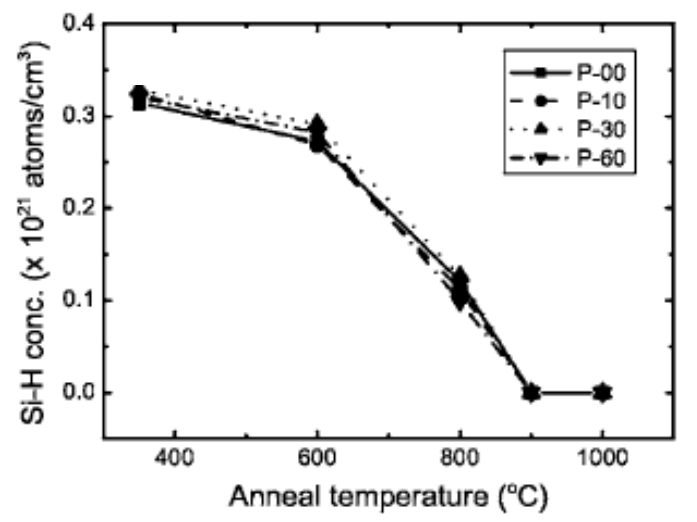

d)

Figure 4: Effect of phosphorus doping in silicon oxynitride: hydrogen content vs. phosphorus flow in the deposition process (a), optical loss spectrum of undoped and P-doped SiON waveguides after annealing at $1000^{\circ} \mathrm{C}(\mathrm{b}), \mathrm{N}-\mathrm{H}$ (c) and Si-H (d) reduction upon annealing in P-doped SiON films. 


\section{CONCLUSION}

We have discussed the high potential of SiON technology for integrated optical devices. The key properties of layers grown by CVD silicon nitride and oxynitride technology are summarized and the performance of selected ring-resonator devices has been shown. One of the key issues for application of SiON technology in telecom devices, reduction of hydrogen incorporation, has been addressed. Preliminary results have shown successful elimination of hydrogen-induced optical loss in phosphorus doped SiON waveguides.

\section{REFERENCES}

[1] K. Wörhoff, et al.: Design and Application of Compact and Highly Tolerant Polarization-Independent Waveguides, J. Lightwave Technol., vol. 25, pp. 1276-1282, 2007

[2] R. Germann, et al.: Silicon oxynitride layers for optical waveguide applications,” J. Electrochem. Soc., vol. 147, pp. 2237-2241, 2000.

[3] M. Hoffmann, et al.: Low-loss fiber-matched low temperature PECVD waveguides with small core dimensions for optical communication systems, IEEE Photon. Technol. Lett., vol. 9, pp. 1238, 1997.

[4] B. E. Little, et al.: Passive components- Very high-order microring resonator filters for WDM applications, IEEE Photon. Technol. Lett., vol. 16, pp. 2263-2265, 2004.

[5] A. Ymeti, et al.: Fast ultrasensitive virus detection using a Young interferometer, Nano Lett., 2006.

[6] L. Zhuang, et al.: Single-chip ring resonator-based $1 \times 8$ optical beam forming network in CMOScompatible waveguide technology, IEEE Photon. Technol. Lett., vol. 19, pp. 1130-1132, 2007.

[7] D.J.W. Klunder, et al.: Vertically and laterally waveguide-coupled cylindrical microresonators in $\mathrm{Si}_{3} \mathrm{~N}_{4}$ on $\mathrm{SiO}_{2}$ technology, Appl. Phys. B, vol. 73, pp. 603-608, 2001.

[8] D.H. Geuzebroek, et al.: , Photonics Technol. Lett., vol. 17, pp.336-338, 2005.

[9] E.J. Klein, et al.: Densely integrated microring resonator based photonic devices for use in access networks, Optics express, vol. 15, pp. 10346-10355, 2007.

[10] K. Wörhoff, et al.: Plasma enhanced chemical vapor deposition of silicon oxynitride optimized for application in integrated optics, Sensors \& Actuators A, vol. 74, pp. 9-12, 1999.

[11] G.M. Hussein, et al.: Reduction of hydrogen-induced optical losses of plasma-enhanced chemical vapour deposition silicon oxynitride by phosphorus doping and heat treatment, J. Appl. Phys., vol. 101, pp. 23517 (6), 2007. 